TITLE:

\title{
Three-dimensional photonic crystals based on double-angled etching and wafer-fusion techniques
}

$\operatorname{AUTHOR}(\mathrm{S})$ :

Takahashi, S; Okano, M; Imada, M; Noda, S

\section{CITATION:}

Takahashi, S ... [et al]. Three-dimensional photonic crystals based on double-angled etching and wafer-fusion techniques. APPLIED PHYSICS LETTERS 2006, 89(12): 123106.

\section{ISSUE DATE:}

2006-09-18

URL:

http://hdl.handle.net/2433/50112

\section{RIGHT:}

Copyright 2006 American Institute of Physics. This article may be downloaded for personal use only. Any other use requires prior permission of the author and the American Institute of Physics. 


\title{
Three-dimensional photonic crystals based on double-angled etching and wafer-fusion techniques
}

\author{
Shigeki Takahashi, ${ }^{a)}$ Makoto Okano, Masahiro Imada, and Susumu Noda \\ Department of Electronic Science and Engineering, Kyoto University, Kyoto 615-8510, Japan
}

(Received 8 April 2006; accepted 31 July 2006; published online 18 September 2006)

\begin{abstract}
We propose a method for the realization of three-dimensional photonic crystals by combining a wafer-fusion technique with a two-stage angled etching process. Etching at an angle of $45^{\circ}$ to the substrate creates woodpile (or stacked striped) structures, and artificial defects and light emitters can then be introduced by the wafer-fusion technique, thereby considerably reducing the number of processes required. Here, we determine the required thickness of the photonic crystals by calculating the transmission properties and quality factors of point-defect cavities. Furthermore, we demonstrate the feasibility of angled etching using an inductively coupled plasma-etching system at cryogenic temperatures. (C) 2006 American Institute of Physics. [DOI: 10.1063/1.2355463]
\end{abstract}

There has been considerable interest in the use of photonic crystals (PCs) to manipulate photons. ${ }^{1-19}$ In particular, three-dimensional (3D) PCs can inhibit the propagation of all optical modes, and are suitable for the ultimate control of light. Thus far, several important methods for the fabrication of 3D PCs in semiconductors such as III-V or group IV that operate at optical wavelengths have been reported, including the wafer-fusion approach, ${ }^{3,20}$ the multidirectional etching technique, ${ }^{21}$ and the surface-micromachining approach. ${ }^{22-24}$ The wafer-fusion approach is valuable for introducing not only artificial defects but also light-emitting elements into 3D PCs. The control of light emission using this approach has been recently reported. ${ }^{25}$ The multidirectional etching technique shows promise for the fabrication of 3D PCs by a single process. In this current letter, we propose and investigate the feasibility of combining the wafer-fusion and multidirectional etching techniques. We employ here a two-stage angled etching process that has recently been theoretically investigated, ${ }^{26}$ but has not yet been used to fabricate 3D PCs in practice. A direct laser writing of the structure onto a photoresist has been recently tried ${ }^{27}$ but not transferred to high index semiconductors. Here, we initially theoretically calculate the transmission properties of 3D PCs fabricated by the double-angled etching technique, in order to estimate the required thickness of the PCs. We then evaluate the quality factor $Q$ of a point-defect cavity introduced by the waferfusion approach. Finally, we demonstrate the fabrication of 3D PCs with double-angled etching using an inductively coupled plasma (ICP) etching technique.

The method considered in this work is illustrated in Fig. 1. A complete 3D PC can be constructed by etching dielectric materials, such as silicon and III-V semiconductors, in both the [110] and [1 $1 \overline{1} 0]$ directions-that is, at angles of $45^{\circ}$ to the substrate [Fig. 1(a)]. The basic structure of the resulting 3D PC is the same as the well-known woodpile or stackedstriped structure. ${ }^{3,20,25,28,29}$ Artificial defects and/or light emitters can then be introduced into the 3D PCs using the wafer-fusion technique [Fig. 1(b)]. Initially, we calculated the transmission properties of 3D PCs of finite thickness for the $\Gamma-\mathrm{X}$ (or $z$ ) direction [Fig. 2(a)] using the 3D finitedifference time-domain (FDTD) method, ${ }^{30,31}$ where the holes

${ }^{a)}$ Electronic mail: stakahashi@qoe.kuee.kyoto-u.ac.jp etched at an angle are assumed to have a rectangular shape. The periods $a_{x}$ and $a_{z}$ along the $x$ and $z$ directions are assumed to be equal: $a_{x}=a_{z}=\sqrt{2} a$ for etching angles of $45^{\circ}$, where $a$ is the distance between parallel stripes [Fig. 2(a)]. The period $a_{y}$ along the $y$ direction and the dielectric filling factor are assumed to be $1.25 a$ and 0.30 , respectively. The refractive index of the dielectric material is set at 3.48, which corresponds to that of silicon at $1.5 \mu \mathrm{m}$ wavelength region. Both the upper and lower sides of the 3D PC are assumed to be covered by the same dielectric material. The calculated transmission properties of $x$-polarized and $y$-polarized lights are shown in Figs. 2(b) and 2(c), respectively. Complete photonic-band-gap (PBG) region is found in the range of $0.342 c / a-0.414 c / a$. The polarization dependence is found to be relatively small, and transmittance in the PBG region decreases by $10 \mathrm{~dB} / a_{z}$ period for both polarizations. These analyses indicate that a thickness of $\sim 3 a_{z}$ in the $z$ direction is required for an attenuation of $-30 \mathrm{~dB}$.

Next, we investigated the effect of introducing a pointdefect cavity at the center of the 3D PC. A schematic representation of the point-defect cavity considered here is shown in Fig. 3(a), where the thickness of the defect layer is set at $a_{z} / 4$, and the widths for the $x$ and $y$ directions of the point-

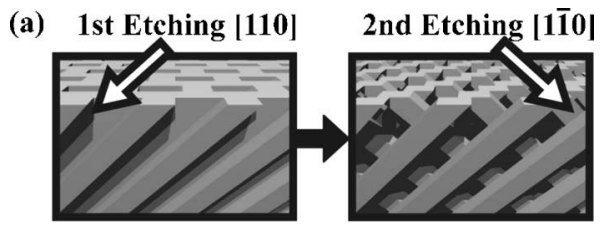

(b)

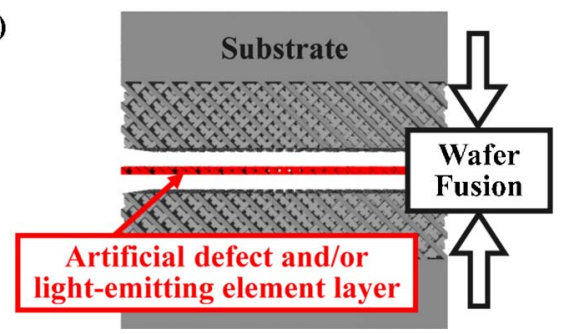

FIG. 1. (Color online) Proposed method for realizing 3D PCs containing artificial light emitters and defects. (a) Double-angled etching process at $45^{\circ}$ to the substrate. (b) Introduction of artificial defects and/or light-emitting elements into 3D PCs by the wafer-fusion method. 
(a)

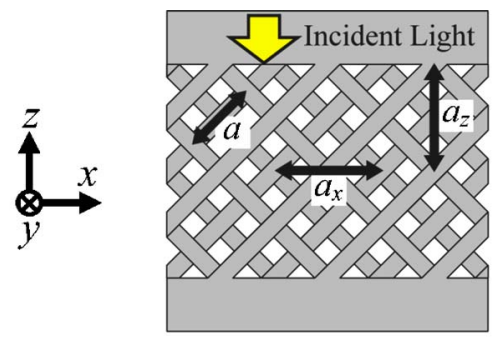

(b)

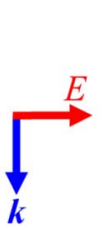

(c)

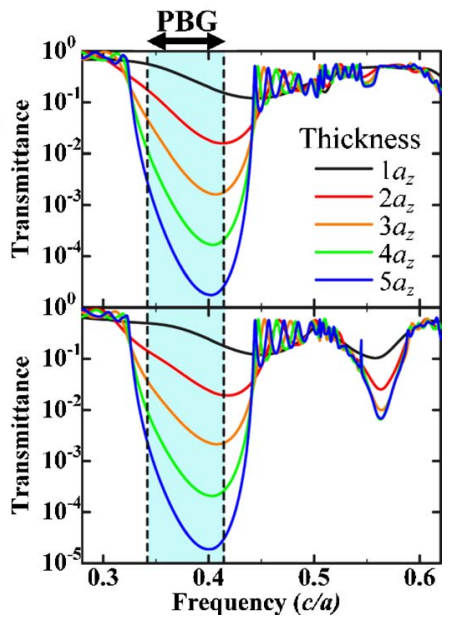

FIG. 2. (Color online) Transmission properties calculated by the 3D FDTD method. (a) Model used in the calculations. The incident light has (b) $x$-axis polarization and (c) $y$-axis polarization. The thickness of the 3D PCs is varied from $a_{z}$ to $5 a_{z}$. The frequency region between $0.342 c / a$ and $0.414 c / a$ corresponds to the complete PBG.

defect cavity are set at $L_{x}=a_{x}$ and $L_{y}=a_{y} / 4$, respectively. We calculated the cavity $Q$ factor of the lowest resonant mode, which has a resonant frequency of $0.389 \mathrm{c} / a$. The dependence of $Q$ on the total thickness of the PC is shown in Fig. 3(b). It is seen that $Q$ increases significantly with thickness,

(a)

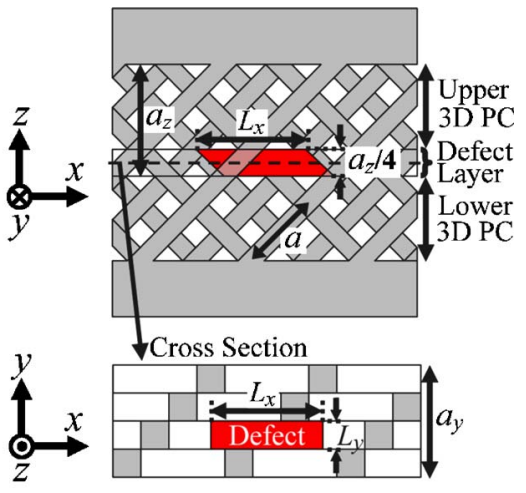

(b)

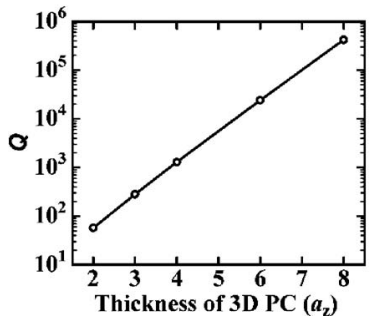

FIG. 3. (Color online) $Q$ factor of a point-defect cavity calculated by the 3D FDTD method. (a) Model used in the calculations. The point-defect cavity is created by adding dielectric material to an oblique rod in the defect layer with thickness $a_{z} / 4$. The point defect can be fabricated by changing the mask pattern of the defect layer. (b) Dependence of the quality factor on 3D PC thickness. The $Q$ factor of the lowest resonant mode is calculated.
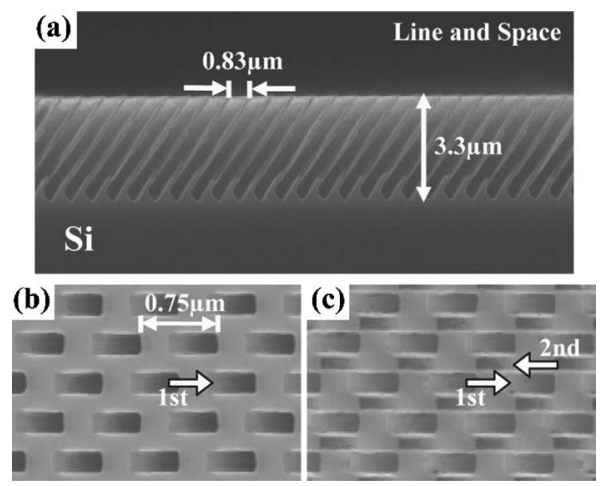

FIG. 4. Scanning electron microscope images of $\mathrm{Si}$ wafers with angled etching. $\mathrm{A} \mathrm{SF}_{6} / \mathrm{O}_{2} \mathrm{ICP}$ etching process at cryogenic temperatures is applied. (a) Cross sectional view of an angled-etched line-and-space pattern. A vertical depth of $3.3 \mu \mathrm{m}$ is achieved. (b) Top view after first angled etching process for fabrication of a 3D PC. (c) Top view of a 3D PC after first and second angled etching processes. The thickness of the $3 \mathrm{DPC}$ is $\sim 1$ period.

and that the use of upper and lower PCs with thicknesses of the order of $2.5 a_{z}$ is sufficient to achieve $Q$ values of several thousands. When we set $a_{x}=a_{z}=0.83 \mu \mathrm{m}$, so as to form a complete PBG at the telecommunications wavelength of $1.55 \mu \mathrm{m}$, the required vertical depth of angled etching is only $\sim 2 \mu \mathrm{m}$.

The realization of a PC with double-angled etching into semiconductors has thus far not been experimentally demonstrated. Therefore, in order to verify the feasibility of the method considered above, we carried out angled plasma etching of $\mathrm{Si}$ wafers, using a $\mathrm{SF}_{6} / \mathrm{O}_{2}$ ICP etching process at cryogenic temperatures. The cryogenic process ${ }^{32}$ is becoming increasingly important in the field of micromachining, because both high selectivity and a high aspect ratio (depthto-width ratio) can be achieved. ${ }^{33}$ We first attempted angled etching to give line-and-space patterns. The widths of the line and space were set at 0.58 and $0.25 \mu \mathrm{m}$, respectively, based on the theoretical calculations described above. A mask pattern was formed by electron-beam lithography. A cross-sectional scanning electron micrograph of the resulting etched Si wafer is shown in Fig. 4(a). An angled etching depth of greater than $3.3 \mu \mathrm{m}$ was attained. We next attempted to fabricate a 3D PC by double-angled etching, using appropriate two-dimensional mask patterns. The results of the first trial are shown in Figs. 4(b) and 4(c). The thickness of the 3D PC is $\sim 1$ period. Preliminary optical measurement results in Fig. 5 showed that transmission had decreased and reflection had increased in the wavelength region

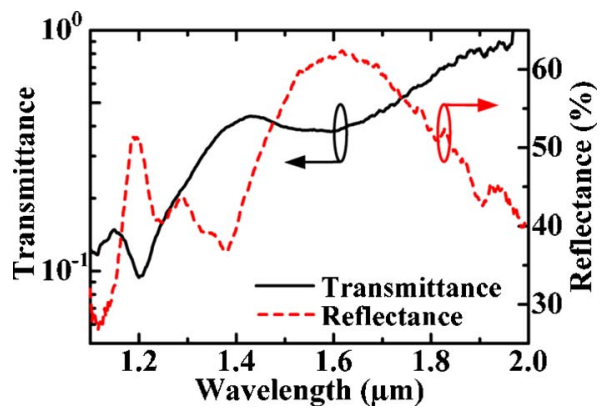

FIG. 5. (Color online) Normal incident transmission (solid line) and reflection (dashed line) spectra for the 3D PC shown in Fig. 4. The references for the transmission and reflection measurements are an area of bare Si adjacent to the 3D PC and Au mirror, respectively. 
around $1.6 \mu \mathrm{m}$, which is consistent with the PBG region estimated from the structural parameters of the sample.

In conclusion, we proposed and investigated the feasibility of a method of fabricating 3D PCs by combining doubleangled etching and wafer-fusion techniques. We showed that the required thickness of the resulting $3 \mathrm{D}$ PC was $\sim 3 a_{z}$ to achieve $30 \mathrm{~dB}$ attenuation. The $Q$ factor of a point-defect cavity was theoretically estimated to be of the order of several thousands when the defect layer was sandwiched on each side by $2.5 a_{z}$ 3D PCs using the wafer-fusion technique. We also demonstrated that the double-angled etching of silicon wafers was indeed possible using a cryogenic ICP dryetching method. These results will encourage us to continue to develop fabrication processes for 3D PCs, and to pursue the realization of ultimate light control through future studies.

This work was supported, in part, by Core Research for Evolutional Science and Technology (CREST) program of the Japan Science and Technology Agency (JST), a Grandin-Aid for Scientific Research from the Japan Society for the Promotion of Science (JSPS), the 21st Century Center of Excellence (COE) Program of Kyoto University, and the Information Technology (IT) Program of the Ministry of Education, Culture, Sports, Science, and Technology of Japan (MEXT).

${ }^{1}$ E. Yablonovitch, Phys. Rev. Lett. 58, 2059 (1987).

${ }^{2}$ S. John, Phys. Today 44(5), 32 (1991).

${ }^{3}$ S. Noda, K. Tomoda, N. Yamamoto, and A. Chutinan, Science 289, 604 (2000).

${ }^{4}$ E. Yablonovitch, T. J. Gmitter, and K. M. Leung, Phys. Rev. Lett. 67, 2295 (1991).

${ }^{5}$ S. G. Romanov, T. Maka, C. M. Sotomayor Torres, M. Müller, and R. Zentel, J. Appl. Phys. 91, 9426 (2002).

${ }^{6}$ S. Noda, A. Chutinan, and M. Imada, Nature (London) 407, 608 (2000).

${ }^{7}$ O. Painter, R. K. Lee, A. Scherer, A. Yariv, J. D. O’Brien, P. D. Dapkus, and I. Kim, Science 284, 1819 (1999).

${ }^{8}$ S. Noda, M. Yokoyama, M. Imada, A. Chutinan, and M. Mochizuki, Science 293, 1123 (2001).

${ }^{9}$ B. Song, S. Noda, and T. Asano, Science 300, 1537 (2003).

${ }^{10}$ Y. Akahane, T. Asano, B. Song, and S. Noda, Nature (London) 425, 944 (2003).

${ }^{11}$ M. Fujita, S. Takahashi, Y. Tanaka, T. Asano, and S. Noda, Science 308, 1296 (2005).

${ }^{12}$ T. Baba, N. Fukaya, and J. Yonekura, Electron. Lett. 35, 654 (1999).

${ }^{13}$ T. D. Happ, M. Kamp, and A. Forchel, Opt. Lett. 26, 1102 (2001).

${ }^{14}$ P. Kramper, M. Agio, C. M. Soukoulis, A. Birner, F. Müller, R. B. Wehrspohn, U. Gösele, and V. Sandoghdar, Phys. Rev. Lett. 92, 113903 (2004).

${ }^{15}$ T. F. Krauss, R. M. De La Rue, and S. Brand, Nature (London) 383, 699 (1996).

${ }^{16}$ M. Notomi, A. Shinya, K. Yamada, J. Takahashi, C. Takahashi, and I. Yokohama, IEEE J. Quantum Electron. 38, 736 (2002).

${ }^{17}$ S. J. McNab, N. Moll, and Y. A. Vlasov, Opt. Express 11, 2927 (2003).

${ }^{18}$ H. Benisty, C. Weisbuch, D. Labilloy, M. Rattier, C. J. M. Smith, T. F. Krauss, R. M. De La Rue, R. Houdré, U. Oesterle, C. Jouanin, and D. Cassagne, J. Lightwave Technol. 17, 2063 (1999).

${ }^{19}$ I. Bulu, H. Caglayan, and E. Ozbay, Phys. Rev. B 67, 205103 (2003).

${ }^{20}$ S. Noda, N. Yamamoto, and A. Sasaki, Jpn. J. Appl. Phys., Part 2 35, L909 (1996).

${ }^{21}$ C. C. Cheng and A. Scherer, J. Vac. Sci. Technol. B 13, 2696 (1995).

${ }^{22}$ S. Y. Lin, J. G. Fleming, D. L. Hetherington, B. K. Smith, R. Biswas, K. M. Ho, M. M. Sigalas, W. Zubrzycki, S. R. Kurtz, and J. Bur, Nature (London) 394, 251 (1998).

${ }^{23}$ J. G. Fleming and S. Y. Lin, Opt. Lett. 24, 49 (1999).

${ }^{24}$ M. Qi, E. Lidorikis, P. T. Rakich, S. G. Johnson, J. D. Joannopoulos, E. P. Ippen, and H. I. Smith, Nature (London) 429, 538 (2004).

${ }^{25}$ S. Ogawa, M. Imada, S. Yoshimono, M. Okano, and S. Noda, Science 305, 227 (2004)

${ }^{26}$ O. Toader, M. Berciu, and S. John, Phys. Rev. Lett. 90, 233901 (2003).

${ }^{27}$ M. Deubel, M. Wegener, A. Kaso, and S. John, Appl. Phys. Lett. 85, 1895 (2004).

${ }^{28}$ K. M. Ho, C. T. Chan, C. M. Soukoulis, R. Biswas, and M. Sigalas, Solid State Commun. 89, 413 (1994).

${ }^{29}$ H. S. Sözüer and J. P. Dowling, J. Mod. Opt. 41, 231 (1994).

${ }^{30}$ M. Okano, A. Chutinan, and S. Noda, Phys. Rev. B 66, 165211 (2002).

${ }^{31}$ M. Okano and S. Noda, Phys. Rev. B 70, 125105 (2004).

${ }^{32}$ S. Tachi, K. Tsujimoto, and S. Okudaira, Appl. Phys. Lett. 52, 616 (1988).

${ }^{33}$ M. J. Walker, Proc. SPIE 4407, 89 (2001). 